\title{
Dietary phytoestrogens and health - a population study
}

\author{
G. G. C. Kuhnle ${ }^{1,2}$, A. Vogiatzoglou ${ }^{1}$, H. A. Ward ${ }^{2}$ and K.-T. Khaw ${ }^{2}$ \\ ${ }^{1}$ Department of Food and Nutritional Sciences, Whiteknights, University of Reading, RG6 6AP, UK and \\ ${ }^{2}$ MRC Centre for Nutritional Epidemiology in Cancer Prevention and Survival, University of Cambridge, CB1 8RN
}

Phytoestrogens are polyphenolic secondary plant metabolites, which have received attention because of their potentially beneficial health effects. In addition to other polyphenolic compounds, phytoestrogens have structural and functional similarities with $17 \beta$-oestradiol and can bind to the oestrogen receptor. Although these compounds have only weak oestrogenic activity $\left(10^{-2}-10^{-3}\right.$ compared with $17 \beta$-oestradiol), they are present in much higher concentration and compete at the receptor complex. Their anti-oestrogenic activity has raised the possibility that these compounds might be beneficial in the prevention of hormone related diseases, such as breast and prostate cancer, as well as osteoporosis, and for the alleviation of menopausal symptoms. Several observational studies have been conducted to investigate the health effects of phytoestrogens, however, results so far have been inconclusive. Whereas studies conducted in Asian populations with high habitual phytoestrogen intake (e.g. $38 \mathrm{mg} / \mathrm{d}$ of soy isoflavones in the Shanghai Women's Health Study ${ }^{(1)}$ ) have shown a protective effect against breast cancer and a positive effect on bone density, most studies conducted in Western populations with lower habitual phytoestrogen intake failed to show an effect ${ }^{(2)}$.

Using a newly developed comprehensive food composition database ${ }^{(3-6)}$, including for the first time the phytoestrogen content of foods of animal origin ${ }^{(3)}$, we have investigated associations between dietary isoflavones, lignans, enterolignans and total phytoestrogens, and health in nested case control samples drawn from EPIC Norfolk, a cohort of 25000 men and women in 1993-2008 ${ }^{(7)}$. No significant associations between phytoestrogen intake and breast cancer were found, neither with urinary biomarkers nor using dietary data ${ }^{(2)}$. Similarly, no significant associations have been observed for colorectal cancer, although in men a marginally significant positive association was observed for the lignan secoisiolariciresinol ${ }^{(7)}$. Risk for prostate cancer was positively associated with intake of enterolignans, compounds found mainly in dairy products ${ }^{(3)}$. However, this association became non-significant after adjusting for Ca as surrogate marker of dairy intake.

In post-menopausal women, the association between bone density and phytoestrogen intake was marginally significant for the non-soy isoflavone formononetin and enterolignans, however, the latter became non-significant after adjusting for Ca intake. In the lowest quintile of $\mathrm{Ca}$ intake (less than $570 \mathrm{mg} / \mathrm{d}$ ), a marginally significant association between bone density and soy isoflavones was observed ${ }^{(8)}$.

These results suggest that phytoestrogens have no significant protective effect against cancer or osteoporosis in the general population with low habitual intake (less than $5 \mathrm{mg} / \mathrm{d}$ ).

This research was supported by the Food Standards Agency (T05028) and the MRC.

1. Zhang X, Shu X-O, Li H et al. (2005) Arch Intern Med 165, 1890-1895

2. Ward HA \& Kuhnle GGC (2010) Arch Biochem Biophys 501, 170-175

3. Kuhnle GGC, Dell'Aquila C, Aspinall SM et al. (2008) J Agric Food Chem 56, 10099-10104

4. Kuhnle GGC, Dell'Aquila C, Aspinall SM et al. (2008) J Agric Food Chem 56(16), 7311-7315

5. Kuhnle GGC, Dell'Aquila C, Aspinall SM et al. (2009) Nutr Cancer 61, 302-309

6. Kuhnle GGC, Dell'Aquila C, Aspinall SM et al. (2009) Food Chem 116, 542-554

7. Day N, Oakes S, Luben RN et al. (1999) Br J Cancer 80, 95-103

8. Kuhnle GGC, Ward A, Vogiatzoglou A et al. (2011) Br J Nutr (In the Press). 